Acta Crystallographica Section E

Structure Reports

Online

ISSN 1600-5368

\section{Potassium 4-nitrophenylsulfonate monohydrate}

The title compound, $\mathrm{K}^{+} \cdot \mathrm{C}_{6} \mathrm{H}_{4} \mathrm{NO}_{5} \mathrm{~S}^{-} \cdot \mathrm{H}_{2} \mathrm{O}$, forms a threedimensional polymeric structure with an $\mathrm{O}_{8}$ coordination environment of the $\mathrm{K}^{+}$cation.

\section{Comment}

As part of a programme aimed at developing new aza-BaeyerVilliger reactions, we have examined the use of $\mathrm{N}$-alkyl- $\mathrm{O}$ arylsulfonylhydroxylamines as potential nitrene equivalents (Hoffman \& Buntain, 1988; Hoffman \& Salvador, 1989a, 1991). Attempts have therefore been made to prepare a range of $N$-alkyl- $O$-arylsulfonylhydroxylamines $p-X \mathrm{C}_{6} \mathrm{H}_{4} \mathrm{SO}_{2} \mathrm{NH} R$ by reacting $R \mathrm{NH}_{2}$ with sulfonyl peroxides $p-X \mathrm{C}_{6} \mathrm{H}_{4} \mathrm{SO}_{2} \mathrm{OO}$ $\mathrm{SO}_{2} \mathrm{C}_{6} \mathrm{H}_{4}-p-X$, which in turn are accessible from sulfonyl chlorides $p-X_{6} \mathrm{H}_{4} \mathrm{SO}_{2} \mathrm{Cl}$ by reaction with $t$-BuOOH (Hoffman \& Cadena, 1977; Hoffman \& Belfoure, 1983; Hoffman \& Salvador, 1989b). The title compound, (I), was isolated as a by-product during this synthesis.

Single-crystal X-ray study
$T=120 \mathrm{~K}$
Mean $\sigma(\mathrm{C}-\mathrm{C})=0.004 \AA$
$R$ factor $=0.042$
$W R$ factor $=0.095$
Data-to-parameter ratio $=14.7$

For details of how these key indicators were automatically derived from the article, see http://journals.iucr.org/e.
(C) 2006 International Union of Crystallography All rights reserved

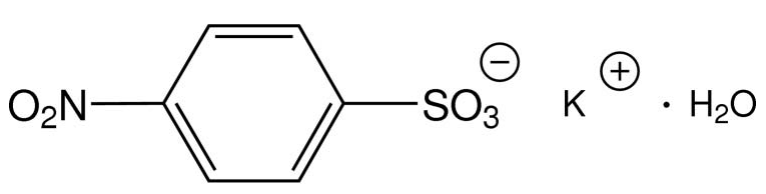

(I)

Numerous esters of 4-nitrophenylsulfonic acid have been structurally characterized, as well as some salts with organic cations (Russell et al., 1994; Chan \& Wong, 2002; Tamura et al., 2002). However, no salt or complex of any metal with this anion has been studied previously.

(I) has a three-dimensional polymeric (catena) crystal structure (Fig. 1). The asymmetric unit comprises one formula unit. The potassium cation is coordinated by eight $\mathrm{O}$ atoms, $v i z$. five from the sulfonate groups of four different anions, one from a nitro group of another anion, and two $\mu_{2}$-bridging water molecules. The coordination polyhedron can be described as a distorted monocapped pentagonal bipyramid. The anion links five $\mathrm{K}^{+}$cations, four of them via one $\mathrm{O}$ atom each. There is only one case of chelation, the sulfonate atoms $\mathrm{O} 1$ and $\mathrm{O} 2$ coordinated to the same potassium ion, and even this one is highly asymmmetric. The $\mathrm{K}-\mathrm{O} 2$ distance is $0.33 \AA$ longer than $\mathrm{K}-\mathrm{O} 1$ and is by far the longest in the structure.

The aqua bridge is highly asymmetric: the $\mathrm{K}-\mathrm{O}$ distances differ by $0.176 \AA$ and the stronger-bound potassium ion is practically coplanar with the $\mathrm{H}_{2} \mathrm{O}$ plane. The weakly coordinated $\mathrm{O} 2$ atom and the uncoordinated $\mathrm{O} 5$ atom of the nitro group act as acceptors of hydrogen bonds donated by the water molecules. Notwithstanding these differences, both $\mathrm{N}-$
Received 19 February 2006 Accepted 3 March 2006 


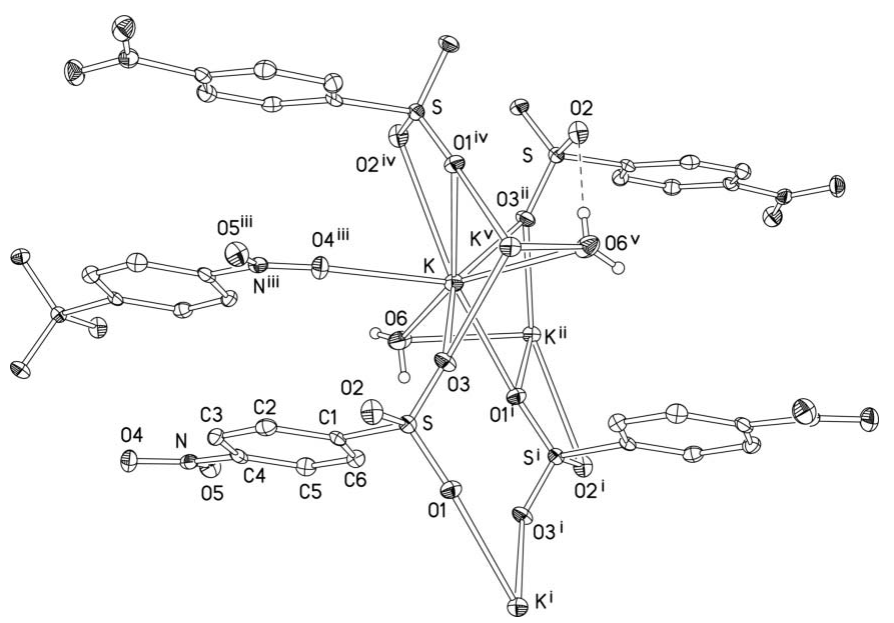

Figure 1

The environment of a $\mathrm{K}^{+}$cation in the structure of (I) (50\% displacement ellipsoids). [Symmetry codes: (i) $1-x, 1-y, 1-z$, (ii) $1-x, \frac{1}{2}+y, \frac{3}{2}-z$, (iii) $-x, 1-y, 1-z$, (iv) $x, \frac{1}{2}-y, \frac{1}{2}+z$, (v) $1-x, y-\frac{1}{2}, \frac{3}{2}-z$.]

$\mathrm{O}$ bond lengths are equal within experimental error (mean 1.233 (3) $\AA$ ), as are the three $\mathrm{S}-\mathrm{O}$ bond lengths (mean 1.453 (3) $\AA$ ). The benzene ring and the nitro group of the anion form a dihedral angle of $11.2(1)^{\circ}$, whereas the $\mathrm{S}-\mathrm{O} 2$ bond is nearly coplanar with the ring: the dihedral angle $\mathrm{C} 2-$ $\mathrm{C} 1-\mathrm{S}-\mathrm{O} 2$ is $9.0(3)^{\circ}$.

\section{Experimental}

4-Nitrobenzenesulfonyl peroxide $p-\mathrm{O}_{2} \mathrm{NC}_{6} \mathrm{H}_{4} \mathrm{SO}_{2} \mathrm{OOSO}_{2} \mathrm{C}_{6} \mathrm{H}_{4} \mathrm{NO}_{2}-p$ (II) was prepared according to Dannley et al. (1970). To a solution of $\mathrm{K}_{2} \mathrm{CO}_{3}(5.10 \mathrm{~g}, 36.9 \mathrm{mmol})$ in water $(76 \mathrm{ml})$, ethanol $(38 \mathrm{ml})$ and hydrogen peroxide $(35 \%, 8.75 \mathrm{~g})$ at $253 \mathrm{~K}$ a cooled $(253 \mathrm{~K})$ solution of 4-nitrobenzenesulfonyl chloride $(7.88 \mathrm{~g}, 35.6 \mathrm{mmol})$ in chloroform $(10 \mathrm{ml})$ was added and the suspension was mixed at full power for $1 \mathrm{~min}$ using a Breville Classique ${ }^{\mathrm{TM}}$ blender. Ethanol $(80 \mathrm{ml})$ was added and the solution was mixed for $4 \mathrm{~min}$ at low power. The precipitate formed was filtered off, washed with distilled water and recrystallized from acetone to give (II) as a yellow solid (2.22 g, 31\%). The filtrate was cooled at $253 \mathrm{~K}$ for $24 \mathrm{~h}$, yielding (I) as yellow crystals $(0.160 \mathrm{~g}, 1.2 \%)$, m.p. $>593 \mathrm{~K}, \mathrm{IR}, v, \mathrm{~cm}^{-1}: 3065$ (CH aromatic stretch), $1529\left(\mathrm{NO}_{2}\right), 1461\left(\mathrm{SO}_{2}\right) 819$ (p-disubstituted aromatic). ${ }^{1} \mathrm{H}$ NMR $\left(200 \mathrm{MHz}, \mathrm{CDCl}_{3}\right): 8.20(d, 2 \mathrm{H}, \mathrm{CH}$ aromatic, $J 8.6 \mathrm{~Hz}), 8.49(d$, $2 \mathrm{H}, \mathrm{CH}$ aromatic, $J=8.4 \mathrm{~Hz}) .{ }^{13} \mathrm{C}$ NMR $(100 \mathrm{MHz}, \mathrm{CDCl} 3): 123.5(2$ $\left.\times \mathrm{PhCNO}_{2}\right), 126.0(4 \times \mathrm{CH}$ aromatic $), 140.0(4 \times \mathrm{CH}$ aromatic $)$, $148.0\left(2 \times \mathrm{PhCSO}_{2}\right)$. The properties of (I) agree with those reported by Kozlov \& Davydov (1965) or Dietze et al. (1989).

\section{Crystal data}

$\mathrm{K}^{+} \cdot \mathrm{C}_{6} \mathrm{H}_{4} \mathrm{NO}_{5} \mathrm{~S}^{-} \cdot \mathrm{H}_{2} \mathrm{O}$
$M_{r}=259.28$
Monoclinic, $P 2_{1} / c$
$a=10.794(1) \AA$
$b=7.1516(6) \AA$
$c=12.417(1) \AA$
$\beta=106.15(1)^{\circ}$
$V=920.70(14) \AA^{3}$
$Z=4$

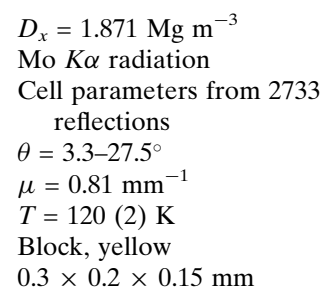

\section{Data collection}

Siemens SMART 1K CCD area detector diffractometer $\omega$ scans

Absorption correction: none

9859 measured reflections

2117 independent reflections

Refinement

Refinement on $F^{2}$

$R\left[F^{2}>2 \sigma\left(F^{2}\right)\right]=0.042$

$w R\left(F^{2}\right)=0.095$

$S=1.05$

2117 reflections

144 parameters

$\mathrm{H}$ atoms treated by a mixture of independent and constrained refinement

Table 1

Selected geometric parameters $\left(\AA{ }^{\circ}{ }^{\circ}\right)$.

\begin{tabular}{|c|c|c|c|}
\hline $\mathrm{K}-\mathrm{O} 1^{\mathrm{i}}$ & $2.712(2)$ & $\mathrm{K}-\mathrm{O} 4^{\mathrm{iii}}$ & $2.802(2)$ \\
\hline $\mathrm{K}-\mathrm{O} 3^{\mathrm{ii}}$ & $2.765(2)$ & $\mathrm{K}-\mathrm{O} 1^{\mathrm{iv}}$ & $2.819(2)$ \\
\hline $\mathrm{K}-\mathrm{O} 3$ & $2.775(2)$ & $\mathrm{K}-\mathrm{O} 6^{\mathrm{v}}$ & $2.955(3)$ \\
\hline $\mathrm{K}-\mathrm{O} 6$ & $2.779(2)$ & $\mathrm{K}-\mathrm{O} 2^{\mathrm{iv}}$ & $3.148(2)$ \\
\hline $\mathrm{O} 1^{\mathrm{i}}-\mathrm{K}-\mathrm{O} 3^{\mathrm{ii}}$ & $87.93(6)$ & $\mathrm{O} 4^{\mathrm{iii}}-\mathrm{K}-\mathrm{O} 1^{\mathrm{iv}}$ & $72.37(6)$ \\
\hline $\mathrm{O} 1^{\mathrm{i}}-\mathrm{K}-\mathrm{O} 3$ & $78.99(6)$ & $\mathrm{O} 1^{\mathrm{i}}-\mathrm{K}-\mathrm{O} 6^{\mathrm{v}}$ & $85.46(7)$ \\
\hline $\mathrm{O} 3^{\mathrm{ii}}-\mathrm{K}-\mathrm{O} 3$ & $151.04(3)$ & $\mathrm{O} 3^{\mathrm{ii}}-\mathrm{K}-\mathrm{O} 6^{\mathrm{v}}$ & $74.70(7)$ \\
\hline $\mathrm{O} 1^{\mathrm{i}}-\mathrm{K}-\mathrm{O} 6$ & $66.46(7)$ & $\mathrm{O} 3-\mathrm{K}-\mathrm{O}^{\mathrm{v}}$ & $78.56(7)$ \\
\hline $\mathrm{O} 3^{\mathrm{ii}}-\mathrm{K}-\mathrm{O} 6$ & $81.82(7)$ & $\mathrm{O} 6-\mathrm{K}-\mathrm{O}^{\mathrm{v}}$ & $143.93(7)$ \\
\hline $\mathrm{O} 3-\mathrm{K}-\mathrm{O} 6$ & $115.27(7)$ & $\mathrm{O} 4^{\mathrm{iii}}-\mathrm{K}-\mathrm{O}^{\mathrm{v}}$ & $127.38(7)$ \\
\hline $\mathrm{O} 1^{\mathrm{i}}-\mathrm{K}-\mathrm{O} 4^{\mathrm{iii}}$ & $128.10(7)$ & $\mathrm{O} 1^{\mathrm{iv}}-\mathrm{K}-\mathrm{O}^{\mathrm{v}}$ & $62.76(7)$ \\
\hline $\mathrm{O} 3^{\mathrm{ii}}-\mathrm{K}-\mathrm{O} 4^{\mathrm{iii}}$ & $134.60(7)$ & $\mathrm{O} 1^{\mathrm{i}}-\mathrm{K}-\mathrm{O} 2^{\mathrm{iv}}$ & $156.06(6)$ \\
\hline $\mathrm{O} 3-\mathrm{K}-\mathrm{O} 4^{\mathrm{iii}}$ & $71.80(7)$ & $\mathrm{O} 3^{\mathrm{ii}}-\mathrm{K}-\mathrm{O} 2^{\mathrm{iv}}$ & $70.29(6)$ \\
\hline $\mathrm{O} 6-\mathrm{K}-\mathrm{O} 4^{\mathrm{iii}}$ & $88.53(7)$ & $\mathrm{O} 3-\mathrm{K}-\mathrm{O} 2^{\mathrm{iv}}$ & $124.93(6)$ \\
\hline $\mathrm{O} 1^{\mathrm{i}}-\mathrm{K}-\mathrm{O} 1^{\mathrm{iv}}$ & $146.95(5)$ & $\mathrm{O} 6-\mathrm{K}-\mathrm{O} 2^{\mathrm{iv}}$ & $99.66(7)$ \\
\hline $\mathrm{O} 3^{\mathrm{ii}}-\mathrm{K}-\mathrm{O} 1^{\mathrm{iv}}$ & $91.85(6)$ & $\mathrm{O} 4^{\mathrm{iii}}-\mathrm{K}-\mathrm{O} 2^{\mathrm{iv}}$ & $67.84(6)$ \\
\hline $\mathrm{O} 3-\mathrm{K}-\mathrm{O} 1^{\mathrm{iv}}$ & $85.63(6)$ & $\mathrm{O} 1^{\mathrm{iv}}-\mathrm{K}-\mathrm{O} 2^{\mathrm{iv}}$ & $47.52(6)$ \\
\hline $\mathrm{O} 6-\mathrm{K}-\mathrm{O} 1^{\mathrm{iv}}$ & $146.11(7)$ & $\mathrm{O} 6^{\mathrm{v}}-\mathrm{K}-\mathrm{O} 2^{\mathrm{iv}}$ & $97.73(7)$ \\
\hline
\end{tabular}

Table 2

Hydrogen-bond geometry $\left(\AA{ }^{\circ}\right)$.

\begin{tabular}{lllll}
\hline$D-\mathrm{H} \cdots A$ & $D-\mathrm{H}$ & $\mathrm{H} \cdots A$ & $D \cdots A$ & $D-\mathrm{H} \cdots A$ \\
\hline $\mathrm{O} 6-\mathrm{H} 01 \cdots \mathrm{O} 2^{\text {vi }}$ & $0.78(4)$ & $2.15(4)$ & $2.922(3)$ & $172(4)$ \\
$\mathrm{O} 6-\mathrm{H} 02 \cdots 5^{\text {vii }}$ & $0.85(4)$ & $2.23(4)$ & $3.050(3)$ & $161(4)$ \\
\hline
\end{tabular}

Symmetry codes: (vi) $x, y+1, z ;$ (vii) $-x,-y+2,-z+1$.

Water atoms H01 and H02 were located in a difference map and refined isotropically. Benzene $\mathrm{H}$ atoms were treated as riding on the C atoms, C $-\mathrm{H} 0.95 \AA$ A $U_{\text {iso }}(\mathrm{H})=1.2 U_{\text {eq }}(\mathrm{C})$.

Data collection: SMART (Bruker, 2001); cell refinement: SAINT (Bruker, 2001); data reduction: $S A I N T$; $\operatorname{program}(\mathrm{s})$ used to solve structure: SHELXTL (Bruker, 2001); program(s) used to refine structure: SHELXTL; molecular graphics: SHELXTL; software used to prepare material for publication: SHELXTL.

We thank both the EPSRC and GlaxoSmithKline Pharmaceuticals for a CASE award (to AJB).

\section{References}

Bruker (2001). SMART (Version 5.625), SAINT (Version 6.02A) and SHELXTL (Version 6.12). Bruker AXS Inc., Madison, Wisconsin, USA. 
Chan, K. W. Y. \& Wong, W. T. (2002). Acta Cryst. E58, o1048-o1050.

Dannley, R. L., Gagen, J. E. \& Stewart, O. J. (1970). J. Org. Chem. 35, 30763079.

Dietze, P. E.; Hariri R. \& Khattak, J. (1989). J. Org. Chem. 54, 33173320.

Hoffman, R. V. \& Belfoure, E. L. (1983). Synthesis, pp. 34-35.

Hoffman, R. V. \& Buntain, G. A. (1988). J. Org. Chem. 53, 3316-3321.

Hoffman, R. V. \& Cadena, R. (1977). J. Am. Chem. Soc. 99, 8226-8232.

Hoffman, R. V. \& Salvador, J. (1989a). Tetrahedron Lett. 30, 4207-4210.
Hoffman, R. V. \& Salvador, J. (1989b). J. Chem. Soc. Perkin Trans. 1, pp. 13751380.

Hoffman, R. V. \& Salvador, J. (1991). Tetrahedron Lett. 32, 2429-2432.

Kozlov V. V. \& Davydov, A. A. (1965). Zh. Org. Khim. 1, 559-562. (In Russian.)

Russell, V. A., Etter, M. C. \& Ward, M. D. (1994). Chem. Mater. 6, 1206-1217.

Tamura, R., Fujimoto, D., Lepp, Z., Misaki, K., Miura, H., Takahashi, H., Ushio, T., Nakai, T. \& Hirotsu, K. (2002). J. Am. Chem. Soc. 124, 1313913153 . 


\section{supporting information}

Acta Cryst. (2006). E62, m741-m743 [https://doi.org/10.1107/S1600536806007835]

\section{Potassium 4-nitrophenylsulfonate monohydrate}

\section{Alexandrea J. Blatch, Judith A. K. Howard, Michael R. Probert, Christian A. Smethurst and Andrew Whiting}

Potassium 4-nitrophenylsulfonate monohydrate

Crystal data

$\mathrm{K}^{+} \cdot \mathrm{C}_{6} \mathrm{H}_{4} \mathrm{NO}_{5} \mathrm{~S}^{-} \cdot \mathrm{H}_{2} \mathrm{O}$

$F(000)=528$

$M_{r}=259.28$

Monoclinic, $P 2_{1} / c$

Hall symbol: -P $2 \mathrm{ybc}$

$D_{\mathrm{x}}=1.871 \mathrm{Mg} \mathrm{m}^{-3}$

$a=10.794$ (1) $\AA$

$b=7.1516(6) \AA$

$c=12.417(1) \AA$

Mo $K \alpha$ radiation, $\lambda=0.71073 \AA$

$\beta=106.15(1)^{\circ}$

Cell parameters from 2733 reflections

$\theta=3.3-27.5^{\circ}$

$\mu=0.81 \mathrm{~mm}^{-1}$

$T=120 \mathrm{~K}$

$V=920.70(14) \AA^{3}$

Block, colourless

$Z=4$

$0.3 \times 0.2 \times 0.15 \mathrm{~mm}$

\section{Data collection}

Siemens SMART 1K CCD area detector diffractometer

Radiation source: fine-focus sealed tube

2117 independent reflections

Graphite monochromator

Detector resolution: 8 pixels $\mathrm{mm}^{-1}$

$\omega$ scans

9859 measured reflections 1596 reflections with $I>2 \sigma(I)$

$R_{\text {int }}=0.067$

$\theta_{\max }=27.5^{\circ}, \theta_{\min }=2.0^{\circ}$

$h=-14 \rightarrow 13$

$k=-9 \rightarrow 9$

$l=-16 \rightarrow 15$

\section{Refinement}

Refinement on $F^{2}$

Least-squares matrix: full

$R\left[F^{2}>2 \sigma\left(F^{2}\right)\right]=0.042$

$w R\left(F^{2}\right)=0.095$

$S=1.05$

2117 reflections

144 parameters

0 restraints

Primary atom site location: structure-invariant direct methods

Secondary atom site location: difference Fourier map

Hydrogen site location: inferred from neighbouring sites

$\mathrm{H}$ atoms treated by a mixture of independent and constrained refinement

$w=1 /\left[\sigma^{2}\left(F_{\mathrm{o}}^{2}\right)+(0.0364 P)^{2}+0.9635 P\right]$ where $P=\left(F_{\mathrm{o}}^{2}+2 F_{\mathrm{c}}{ }^{2}\right) / 3$

$(\Delta / \sigma)_{\max }<0.001$

$\Delta \rho_{\max }=0.39 \mathrm{e} \AA^{-3}$

$\Delta \rho_{\min }=-0.46$ e $\AA^{-3}$

Special details

Experimental. The data collection nominally covered full sphere of reciprocal Space, by a combination of 5 sets of $\omega$ scans each set at different $\varphi$ and/or $2 \theta$ angles and each scan (10 s exposure) covering $0.3^{\circ}$ in $\omega$. Crystal to detector distance $4.51 \mathrm{~cm}$. 
Geometry. All e.s.d.'s (except the e.s.d. in the dihedral angle between two 1.s. planes) are estimated using the full covariance matrix. The cell e.s.d.'s are taken into account individually in the estimation of e.s.d.'s in distances, angles and torsion angles; correlations between e.s.d.'s in cell parameters are only used when they are defined by crystal symmetry. An approximate (isotropic) treatment of cell e.s.d.'s is used for estimating e.s.d.'s involving 1.s. planes.

Refinement. Refinement of $F^{2}$ against ALL reflections. The weighted $R$-factor $w R$ and goodness of fit $S$ are based on $F^{2}$, conventional $R$-factors $R$ are based on $F$, with $F$ set to zero for negative $F^{2}$. The threshold expression of $F^{2}>\sigma\left(F^{2}\right)$ is used only for calculating $R$-factors(gt) etc. and is not relevant to the choice of reflections for refinement.

Fractional atomic coordinates and isotropic or equivalent isotropic displacement parameters $\left(\AA^{2}\right)$

\begin{tabular}{lllll}
\hline & $x$ & $y$ & $z$ & $U_{\text {iso }} / U_{\text {eq }}$ \\
\hline K & $0.43521(6)$ & $0.53657(9)$ & $0.74392(5)$ & $0.01343(16)$ \\
S & $0.37624(7)$ & $0.22064(10)$ & $0.46669(6)$ & $0.01054(17)$ \\
O1 & $0.4524(2)$ & $0.2686(3)$ & $0.39107(17)$ & $0.0140(5)$ \\
O2 & $0.3285(2)$ & $0.0291(3)$ & $0.45242(18)$ & $0.0159(5)$ \\
O3 & $0.4386(2)$ & $0.2697(3)$ & $0.58251(17)$ & $0.0149(5)$ \\
O4 & $-0.1962(2)$ & $0.6461(3)$ & $0.31470(18)$ & $0.0192(5)$ \\
O5 & $-0.0704(2)$ & $0.8883(3)$ & $0.35935(18)$ & $0.0188(5)$ \\
O6 & $0.3076(2)$ & $0.8669(3)$ & $0.6626(2)$ & $0.0207(5)$ \\
H01 & $0.315(4)$ & $0.900(5)$ & $0.605(3)$ & $0.026(11)^{*}$ \\
H02 & $0.252(4)$ & $0.945(6)$ & $0.672(3)$ & $0.039(12)^{*}$ \\
N & $-0.0886(2)$ & $0.7181(4)$ & $0.3463(2)$ & $0.0147(5)$ \\
C1 & $0.2366(3)$ & $0.3660(4)$ & $0.4267(2)$ & $0.0108(6)$ \\
C2 & $0.1131(3)$ & $0.2881(4)$ & $0.4060(2)$ & $0.0132(6)$ \\
H2 & 0.1032 & 0.1568 & 0.4118 & $0.016^{*}$ \\
C3 & $0.0058(3)$ & $0.4025(4)$ & $0.3772(2)$ & $0.0136(6)$ \\
H3 & -0.0787 & 0.3518 & 0.3622 & $0.016^{*}$ \\
C4 & $0.0250(3)$ & $0.5950(4)$ & $0.3708(2)$ & $0.0122(6)$ \\
C5 & $0.1463(3)$ & $0.6745(4)$ & $0.3908(2)$ & $0.0144(6)$ \\
H5 & 0.1559 & 0.8060 & 0.3859 & $0.017^{*}$ \\
C6 & $0.2534(3)$ & $0.5582(4)$ & $0.4183(2)$ & $0.0139(6)$ \\
H6 & 0.3376 & 0.6092 & 0.4312 & $0.017^{*}$ \\
& & & &
\end{tabular}

Atomic displacement parameters $\left(\AA^{2}\right)$

\begin{tabular}{lllllll}
\hline & $U^{11}$ & $U^{22}$ & $U^{33}$ & $U^{12}$ & $U^{13}$ & $U^{23}$ \\
\hline $\mathrm{K}$ & $0.0157(3)$ & $0.0116(3)$ & $0.0132(3)$ & $-0.0007(3)$ & $0.0043(2)$ & $0.0000(2)$ \\
$\mathrm{S}$ & $0.0110(4)$ & $0.0094(3)$ & $0.0109(4)$ & $0.0007(3)$ & $0.0023(3)$ & $-0.0001(3)$ \\
$\mathrm{O} 1$ & $0.0159(11)$ & $0.0149(11)$ & $0.0126(11)$ & $0.0009(8)$ & $0.0063(9)$ & $0.0003(8)$ \\
O2 & $0.0161(11)$ & $0.0095(10)$ & $0.0212(12)$ & $0.0010(9)$ & $0.0036(9)$ & $0.0014(9)$ \\
O3 & $0.0169(11)$ & $0.0159(11)$ & $0.0097(10)$ & $0.0022(9)$ & $0.0000(8)$ & $-0.0016(8)$ \\
O4 & $0.0121(12)$ & $0.0241(12)$ & $0.0207(12)$ & $-0.0035(9)$ & $0.0032(9)$ & $0.0018(9)$ \\
O5 & $0.0191(12)$ & $0.0150(11)$ & $0.0217(12)$ & $0.0042(9)$ & $0.0046(10)$ & $0.0011(9)$ \\
O6 & $0.0237(14)$ & $0.0214(13)$ & $0.0197(13)$ & $0.0069(10)$ & $0.0106(11)$ & $0.0055(10)$ \\
N & $0.0137(14)$ & $0.0205(14)$ & $0.0107(13)$ & $0.0018(11)$ & $0.0047(10)$ & $0.0033(10)$ \\
C1 & $0.0109(14)$ & $0.0142(15)$ & $0.0061(14)$ & $-0.0007(12)$ & $0.0003(11)$ & $-0.0015(11)$ \\
C2 & $0.0177(16)$ & $0.0117(14)$ & $0.0110(14)$ & $-0.0015(12)$ & $0.0055(12)$ & $-0.0023(11)$ \\
C3 & $0.0127(15)$ & $0.0168(15)$ & $0.0115(15)$ & $-0.0049(12)$ & $0.0039(12)$ & $-0.0005(11)$ \\
C4 & $0.0126(15)$ & $0.0154(15)$ & $0.0070(14)$ & $0.0023(12)$ & $0.0002(11)$ & $0.0002(11)$
\end{tabular}




\begin{tabular}{lllllll} 
C5 & $0.0175(16)$ & $0.0120(14)$ & $0.0137(15)$ & $0.0002(12)$ & $0.0045(12)$ & $-0.0010(11)$ \\
C6 & $0.0129(15)$ & $0.0164(16)$ & $0.0128(15)$ & $0.0002(12)$ & $0.0041(12)$ & $0.0009(11)$ \\
\hline
\end{tabular}

Geometric parameters $(\AA, \stackrel{\circ}{)}$

\begin{tabular}{|c|c|c|c|}
\hline $\mathrm{K}-\mathrm{O} 1^{\mathrm{i}}$ & $2.712(2)$ & $\mathrm{O} 6-\mathrm{H} 01$ & $0.78(4)$ \\
\hline $\mathrm{K}-\mathrm{O} 3^{\mathrm{ii}}$ & $2.765(2)$ & O6- $-\mathrm{H} 02$ & 0.85 (4) \\
\hline $\mathrm{K}-\mathrm{O} 3$ & $2.775(2)$ & $\mathrm{N}-\mathrm{C} 4$ & $1.471(4)$ \\
\hline $\mathrm{K}-\mathrm{O} 6$ & $2.779(2)$ & $\mathrm{C} 1-\mathrm{C} 6$ & $1.395(4)$ \\
\hline $\mathrm{K}-\mathrm{O} 4^{\mathrm{iii}}$ & $2.802(2)$ & $\mathrm{C} 1-\mathrm{C} 2$ & $1.401(4)$ \\
\hline $\mathrm{K}-\mathrm{O} 1^{\mathrm{iv}}$ & $2.819(2)$ & $\mathrm{C} 2-\mathrm{C} 3$ & $1.382(4)$ \\
\hline $\mathrm{K}-\mathrm{O} 6^{\mathrm{v}}$ & $2.955(3)$ & $\mathrm{C} 2-\mathrm{H} 2$ & 0.9500 \\
\hline $\mathrm{K}-\mathrm{O} 2^{\mathrm{iv}}$ & $3.148(2)$ & $\mathrm{C} 3-\mathrm{C} 4$ & $1.398(4)$ \\
\hline $\mathrm{S}-\mathrm{O} 1$ & $1.451(2)$ & $\mathrm{C} 3-\mathrm{H} 3$ & 0.9500 \\
\hline $\mathrm{S}-\mathrm{O} 3$ & $1.451(2)$ & $\mathrm{C} 4-\mathrm{C} 5$ & $1.384(4)$ \\
\hline $\mathrm{S}-\mathrm{O} 2$ & $1.457(2)$ & $\mathrm{C} 5-\mathrm{C} 6$ & $1.388(4)$ \\
\hline $\mathrm{S}-\mathrm{C} 1$ & $1.784(3)$ & $\mathrm{C} 5-\mathrm{H} 5$ & 0.9500 \\
\hline $\mathrm{O} 4-\mathrm{N}$ & $1.230(3)$ & C6-H6 & 0.9500 \\
\hline $\mathrm{O} 5-\mathrm{N}$ & $1.236(3)$ & & \\
\hline $\mathrm{O} 1^{\mathrm{i}}-\mathrm{K}-\mathrm{O} 3^{\mathrm{ii}}$ & $87.93(6)$ & $\mathrm{S}-\mathrm{O} 1-\mathrm{K}^{\mathrm{vi}}$ & $106.82(10)$ \\
\hline $\mathrm{O} 1 \mathrm{i}-\mathrm{K}-\mathrm{O} 3$ & $78.99(6)$ & $\mathrm{K}^{\mathrm{i}}-\mathrm{O} 1-\mathrm{K}^{\mathrm{vi}}$ & $87.55(6)$ \\
\hline 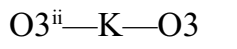 & $151.04(3)$ & $\mathrm{S}-\mathrm{O} 2-\mathrm{K}^{\mathrm{vi}}$ & $92.42(10)$ \\
\hline $\mathrm{O} 1^{\mathrm{i}-\mathrm{K}}-\mathrm{O} 6$ & $66.46(7)$ & $\mathrm{S}-\mathrm{O} 3-\mathrm{K}^{\mathrm{v}}$ & $128.22(11)$ \\
\hline $\mathrm{O} 3^{\mathrm{ii}-\mathrm{K}-\mathrm{O} 6}$ & $81.82(7)$ & $\mathrm{S}-\mathrm{O} 3-\mathrm{K}$ & $141.86(12)$ \\
\hline $\mathrm{O} 3-\mathrm{K}-\mathrm{O} 6$ & $115.27(7)$ & $\mathrm{K}^{\mathrm{v}}-\mathrm{O} 3-\mathrm{K}$ & $87.38(6)$ \\
\hline $\mathrm{O} 1^{\mathrm{i}}-\mathrm{K}-\mathrm{O} 4^{\mathrm{iii}}$ & $128.10(7)$ & $\mathrm{N}-\mathrm{O} 4-\mathrm{K}^{\mathrm{iii}}$ & $175.69(19)$ \\
\hline $\mathrm{O} 3^{\mathrm{ii}-\mathrm{K}}-\mathrm{O} 4^{\mathrm{iii}}$ & $134.60(7)$ & $\mathrm{K}-\mathrm{O} 6-\mathrm{K}^{\mathrm{ii}}$ & $83.67(7)$ \\
\hline $\mathrm{O} 3-\mathrm{K}-\mathrm{O} 4^{\mathrm{iii}}$ & $71.80(7)$ & $\mathrm{K}-\mathrm{O} 6-\mathrm{H} 01$ & $115(3)$ \\
\hline $\mathrm{O} 6-\mathrm{K}-\mathrm{O} 4^{\mathrm{iii}}$ & $88.53(7)$ & $\mathrm{K}^{\mathrm{ii}}-\mathrm{O} 6-\mathrm{H} 01$ & $85(3)$ \\
\hline $\mathrm{O} 1^{\mathrm{i}}-\mathrm{K}-\mathrm{O} 1^{\mathrm{iv}}$ & $146.95(5)$ & $\mathrm{K}-\mathrm{O} 6-\mathrm{H} 02$ & $144(3)$ \\
\hline $\mathrm{O} 3^{\mathrm{ii}-\mathrm{K}}-\mathrm{O} 1^{\mathrm{iv}}$ & $91.85(6)$ & $\mathrm{K}^{\mathrm{ii}}-\mathrm{O} 6-\mathrm{H} 02$ & $108(3)$ \\
\hline $\mathrm{O} 3-\mathrm{K}-\mathrm{O} 1^{\mathrm{iv}}$ & $85.63(6)$ & $\mathrm{H} 01-\mathrm{O} 6-\mathrm{H} 02$ & $100(4)$ \\
\hline $\mathrm{O} 6-\mathrm{K}-\mathrm{O} 1^{\mathrm{iv}}$ & $146.11(7)$ & $\mathrm{O} 4-\mathrm{N}-\mathrm{O} 5$ & $123.7(3)$ \\
\hline $\mathrm{O} 4^{\mathrm{iii}}-\mathrm{K}-\mathrm{O} 1^{\mathrm{iv}}$ & 72.37 (6) & $\mathrm{O} 4-\mathrm{N}-\mathrm{C} 4$ & $118.3(2)$ \\
\hline $\mathrm{O} 1^{\mathrm{i}}-\mathrm{K}-\mathrm{O}^{\mathrm{v}}$ & $85.46(7)$ & $\mathrm{O} 5-\mathrm{N}-\mathrm{C} 4$ & $118.0(2)$ \\
\hline $\mathrm{O} 3^{\mathrm{ii}}-\mathrm{K}-\mathrm{O} 6^{\mathrm{v}}$ & $74.70(7)$ & $\mathrm{C} 6-\mathrm{C} 1-\mathrm{C} 2$ & $121.0(3)$ \\
\hline $\mathrm{O} 3-\mathrm{K}-\mathrm{O}^{\mathrm{v}}$ & $78.56(7)$ & $\mathrm{C} 6-\mathrm{C} 1-\mathrm{S}$ & $118.5(2)$ \\
\hline $\mathrm{O} 6-\mathrm{K}-\mathrm{O}^{\mathrm{v}}$ & $143.93(7)$ & $\mathrm{C} 2-\mathrm{C} 1-\mathrm{S}$ & $120.4(2)$ \\
\hline $\mathrm{O} 4^{\mathrm{iii}}-\mathrm{K}-\mathrm{O}^{\mathrm{v}}$ & $127.38(7)$ & $\mathrm{C} 3-\mathrm{C} 2-\mathrm{C} 1$ & $119.9(3)$ \\
\hline $\mathrm{O} 1^{\mathrm{iv}}-\mathrm{K}-\mathrm{O}^{\mathrm{v}}$ & $62.76(7)$ & $\mathrm{C} 3-\mathrm{C} 2-\mathrm{H} 2$ & 120.0 \\
\hline $\mathrm{O} 1^{\mathrm{i}}-\mathrm{K}-\mathrm{O} 2^{\mathrm{iv}}$ & $156.06(6)$ & $\mathrm{C} 1-\mathrm{C} 2-\mathrm{H} 2$ & 120.0 \\
\hline $\mathrm{O} 3^{\mathrm{ii}}-\mathrm{K}-\mathrm{O} 2^{\mathrm{iv}}$ & $70.29(6)$ & $\mathrm{C} 2-\mathrm{C} 3-\mathrm{C} 4$ & $118.1(3)$ \\
\hline $\mathrm{O} 3-\mathrm{K}-\mathrm{O} 2^{\mathrm{iv}}$ & $124.93(6)$ & $\mathrm{C} 2-\mathrm{C} 3-\mathrm{H} 3$ & 121.0 \\
\hline $\mathrm{O} 6-\mathrm{K}-\mathrm{O} 2^{\mathrm{iv}}$ & $99.66(7)$ & $\mathrm{C} 4-\mathrm{C} 3-\mathrm{H} 3$ & 121.0 \\
\hline $\mathrm{O} 4^{\mathrm{iii}}-\mathrm{K}-\mathrm{O} 2^{\mathrm{iv}}$ & $67.84(6)$ & $\mathrm{C} 5-\mathrm{C} 4-\mathrm{C} 3$ & $122.8(3)$ \\
\hline $\mathrm{O} 1^{\mathrm{iv}}-\mathrm{K}-\mathrm{O} 2^{\mathrm{iv}}$ & $47.52(6)$ & $\mathrm{C} 5-\mathrm{C} 4-\mathrm{N}$ & $118.9(3)$ \\
\hline $\mathrm{O}^{\mathrm{v}}-\mathrm{K}-\mathrm{O} 2^{\mathrm{iv}}$ & $97.73(7)$ & $\mathrm{C} 3-\mathrm{C} 4-\mathrm{N}$ & $118.2(3)$ \\
\hline
\end{tabular}




$\begin{array}{llll}\mathrm{O} 1-\mathrm{S}-\mathrm{O} 3 & 113.22(12) & \mathrm{C} 4-\mathrm{C} 5-\mathrm{C} 6 & 118.7(3) \\ \mathrm{O} 1-\mathrm{S}-\mathrm{O} 2 & 112.87(12) & \mathrm{C} 4-\mathrm{C} 5-\mathrm{H} 5 & 120.7 \\ \mathrm{O} 3-\mathrm{S}-\mathrm{O} 2 & 113.49(13) & \mathrm{C} 6-\mathrm{C} 5-\mathrm{H} 5 & 120.7 \\ \mathrm{O} 1-\mathrm{S}-\mathrm{C} 1 & 105.61(13) & \mathrm{C} 5-\mathrm{C} 6-\mathrm{C} 1 & 119.5(3) \\ \mathrm{O} 3-\mathrm{S}-\mathrm{C} 1 & 104.94(12) & \mathrm{C} 5-\mathrm{C} 6-\mathrm{H} 6 & 120.3 \\ \mathrm{O} 2-\mathrm{S}-\mathrm{C} 1 & 105.73(13) & \mathrm{C} 1-\mathrm{C} 6-\mathrm{H} 6 & 120.3 \\ \mathrm{~S}-\mathrm{O} 1-\mathrm{K}^{\mathrm{i}} & 162.38(12) & & -9.6(4) \\ \mathrm{C} 2-\mathrm{C} 1-\mathrm{S}-\mathrm{O} 2 & 9.0(3) & \mathrm{C} 5-\mathrm{C} 4-\mathrm{N}-\mathrm{O} 5 & \\ \mathrm{C} 3-\mathrm{C} 4-\mathrm{N}-\mathrm{O} 4 & -11.6(4) & & \end{array}$

Symmetry codes: (i) $-x+1,-y+1,-z+1$; (ii) $-x+1, y+1 / 2,-z+3 / 2$; (iii) $-x,-y+1,-z+1$; (iv) $x,-y+1 / 2, z+1 / 2$; (v) $-x+1, y-1 / 2,-z+3 / 2$; (vi) $x,-y+1 / 2$, $z-1 / 2$.

Hydrogen-bond geometry $\left(A,{ }^{\circ}\right)$

\begin{tabular}{lllll}
\hline$D-\mathrm{H} \cdots A$ & $D-\mathrm{H}$ & $\mathrm{H} \cdots A$ & $D \cdots A$ & $D-\mathrm{H} \cdots A$ \\
\hline $\mathrm{O} 6-\mathrm{H} 01 \cdots \mathrm{O} 2^{\text {vii }}$ & $0.78(4)$ & $2.15(4)$ & $2.922(3)$ & $172(4)$ \\
$\mathrm{O} 6-\mathrm{H} 02 \cdots 5^{\text {viii }}$ & $0.85(4)$ & $2.23(4)$ & $3.050(3)$ & $161(4)$ \\
\hline
\end{tabular}

Symmetry codes: (vii) $x, y+1, z$; (viii) $-x,-y+2,-z+1$. 